\title{
dIAP: Knowledge Sharing Amidst the Pandemic
}

\author{
BakUl Jayant Parekh ${ }^{1 *}$ and Arun Bansal ${ }^{2}$ \\ ${ }^{1}$ President and ${ }^{2}$ EB Member, Indian Academy of Pediatrics 2020 \\ *bakulparekh55@gmail.com
}

$\mathrm{T}$ his year has been a year of firsts for the Indian Academy of Pediatrics (IAP). It started two years ago, when you all elected me as the President elect of our mother organization IAP, unopposed - first time in the history of IAP in last four decades. This shows the faith and affection you all have for me, and honestly, that has left me greatly humbled. It motivated me to go the extra mile to achieve the best for our mother organization IAP. I had a vision which would change the way that our medical fraternity works, and benefit our many fellow pediatricians who are generally practicing in the remote locations and rural areas. The dIAP platform - a technology driven academy - was established for the first time. With dIAP we reached the unreached, taking whatever the IAP has to offer to every corner of our country and beyond.

None of us were prepared for what happened next. COVID-19 struck - an epidemic of global proportions! Before the financial year ended, India was forced into a lock-down situation. Academics became a secondary require-ment - survival was of utmost importance. We, the Doctors and the healthcare industry started working overtime, trying to prevent the spread and find a cure, to help people survive. This was the time for IAP to come to the rescue of members and community to continue its academics, social and community activities. No one knew what to do. Desperate times called for desperate measures. We had to start thinking of different ways and so called 'new norms'. Physical distancing, the necessity to use masks, and avoidance of close contact led many to search for 'work from home' options. Likewise, students in various disciplines are being taught by dedicated teachers using the online platform. Medical students, both undergraduates and postgraduates are allowed limited or no bedside learning time to avoid unnecessary exposure. Apart from the routine work done by postgraduates in wards, where they learn practical skills, formal teaching activity has mainly remained restricted. Grand rounds, combined teaching from faculty, and bedside case presentations have come to a halt. Seminars with PowerPoint presentations involving large gatherings in the departments have also been suspended.
Worldwide, it has been recognized that while it is important to provide patient care, it is also necessary to ensure adequate training and teaching of medical students who are future physicians. But the logical and practical concerns of patient safety remain, as these students may act as asymptomatic carriers of SARS-CoV-2 (Severe acute respiratory syndrome - Coronavirus 2). Technology has come to our rescue to continue education through the digital platform. Medical education has transformed through the use of online media for virtual team meetings, clinical skills learning, and even for conducting examinations. Many medical colleges have converted their usual classroom teaching to the elearning platform using various applications available for online classes and webinars. As most of the teaching hospitals cater to a massive burden of patients and most of the teaching staff is involved in clinical work, it allows them lesser time to dedicate for teaching. Also, the senior faculty is actively involved in administrative issues like ensuring preparedness for managing the pandemic efficiently on a large scale.

Keeping these points in mind, the Indian Academy of Pediatrics has rightly introduced digital lectures, by the name of Digital-IAP (dIAP), to facilitate e-learning in all spheres of pediatrics. The dIAP team was already working quite hard. As the pandemic spread, they needed to pull up their socks and ensure that they could deliver well before the expected timeline. I am happy to say that IAP was amongst the first organizations to start the webinar concept on such a large scale. It was and still is hugely popular. People across the country have started viewing and absorbing the knowledge shared in these webinars. Knowledge was not only limited to COVID-19 and other academic topics, but rather a holistic approach was taken to ensure the best interests of our members requirements like teleconsultation solution, health and death insurance, medicolegal support, COVID-19 guidelines, connecting with government, charitable and social responsibilities, and so on. These sessions are being conducted daily, including topics from all sub-specialties and cover the curriculum of postgraduate teaching. dIAP is freely accessible to all, and daily reminders are sent to IAP 
members via social media and registered email IDs. Apart from live streaming, an option to view the recorded version has been made available in the archives. Webcast attendees may actively participate in these sessions, ask queries, and share opinions using the chat box or direct communication in personal meetings.

IAP has conducted 422 online sessions from March 16, 2020 to August 31, 2020, with an overwhelming response in each of these sessions from all over India (Fig. 1). Twenty-three (5\%) sessions were conducted during the morning hours (9 AM to $12 \mathrm{PM}), 278(66 \%)$ sessions in the afternoon (1 PM to 4 PM), and 121 (29\%) sessions in the evening (4 PM onwards). A total of 7,71,375 views with an average of 1886 views per session have been documented. The specialty-wise distribution is shown in Fig. 2. Twentythree $(5 \%)$ of these sessions were directly or indirectly related to COVID-19, and the postgraduate clinics on Thursdays constituted $20(4.7 \%)$ of these sessions. The Pediatric Intensive Care Chapter of IAP has begun the PICU e-Gurukul program, in which weekly lectures are taken by stalwarts in the field of critical care. They have also started P2P PICU Febinar (Peer-to-Peer PICU Fellows' Webinar), providing a national platform for the pediatric intensive care fellows to present, teach and learn from each other. It is a program ' Of the Fellows, By the Fellows, For the Fellows.' The IAP respiratory chapter has started 'Respinars' for pulmonology teaching, in which the experts of the field take lectures.

Similarly, each zone/state wing of IAP has also formulated their teaching/academic sessions online. dIAP has also partnered with the NHS UK through the
Alder Hey academy global pediatric lecture series (GPLS), thereby allowing an opportunity for Indian students and pediatricians to participate in distinguished lectures overseas. IAP has also come up with the unique concept of conducting online conferences through dIAP. The zonal meetings of IAP are being conducted by the name of 'PediWeek'. Each zone was given one week, and this activity was a huge hit and has been appreciated by one and all. It was a mix of scientific and cultural activities. All pediatricians and members of IAP had actively participated in these PediWeeks.

The dIAP sessions have helped in reaching students, private practitioners, and pediatricians at various hospitals, who have benefitted from these classes. The online learning archives could form a database for teaching in the future. There have been some sessions involving parents where they join hands with the pediatricians for the wellbeing of the children. dIAP has brought the teaching to your homes; it is eco-friendly, paperless, saves travel and a lot of time. Moreover, all the sessions are recorded, and one can watch them at their convenience.

However, it also has a few disadvantages inherent to the online platform (Fig. 3). Literature on the impact of the pandemic on online medical education has flagged communication and student assessment problems, technology-related issues, and difficulties in time management in these sessions. Despite these challenges and technophobia, a majority have been able to achieve the expertise quickly to conduct digital classes. Another drawback is that it has decreased the personal touch and

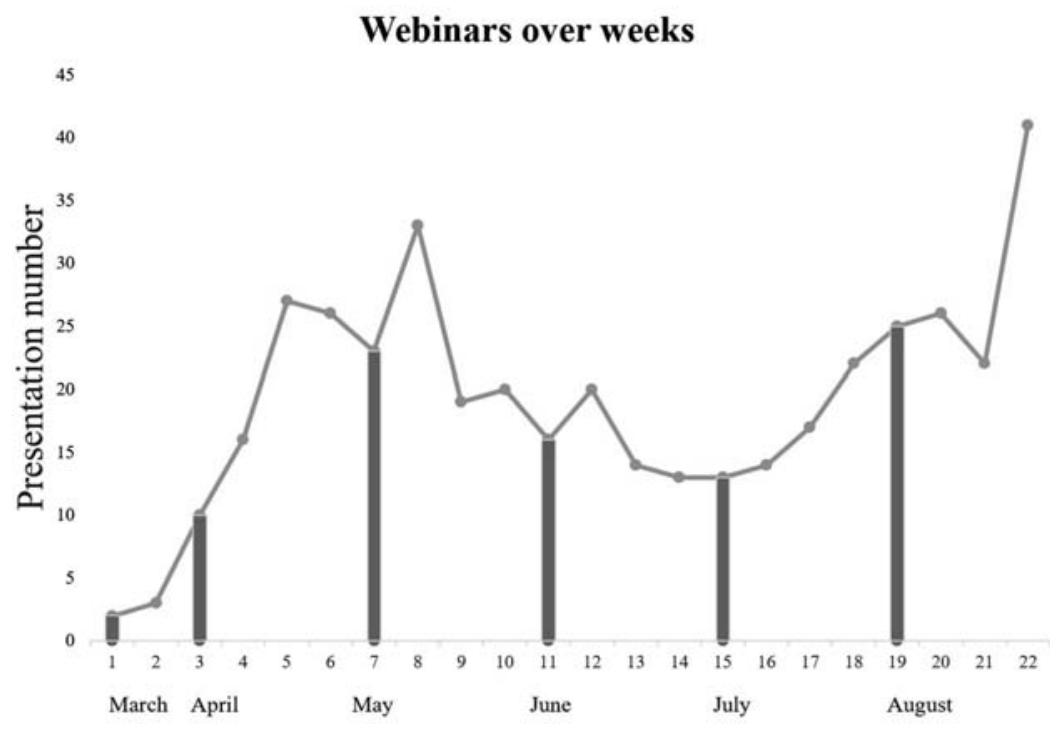

Fig.1 Trend of webinar numbers over the weeks. 


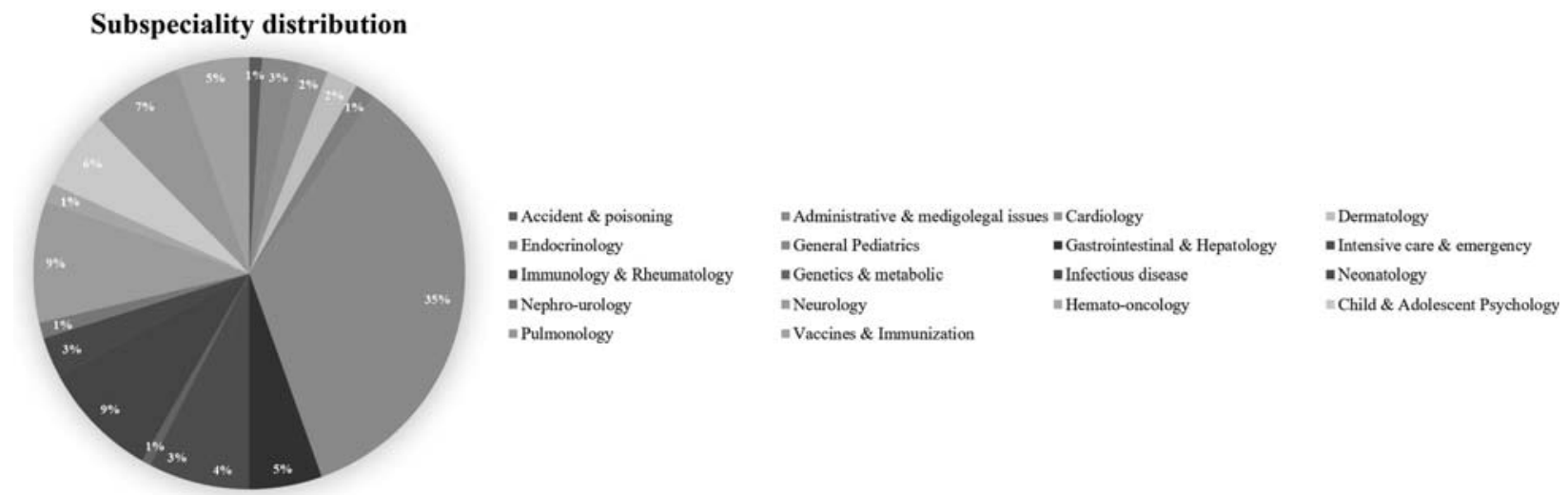

Fig. 2 Sub-specialty distribution of online teaching sessions on the dIAP platform.

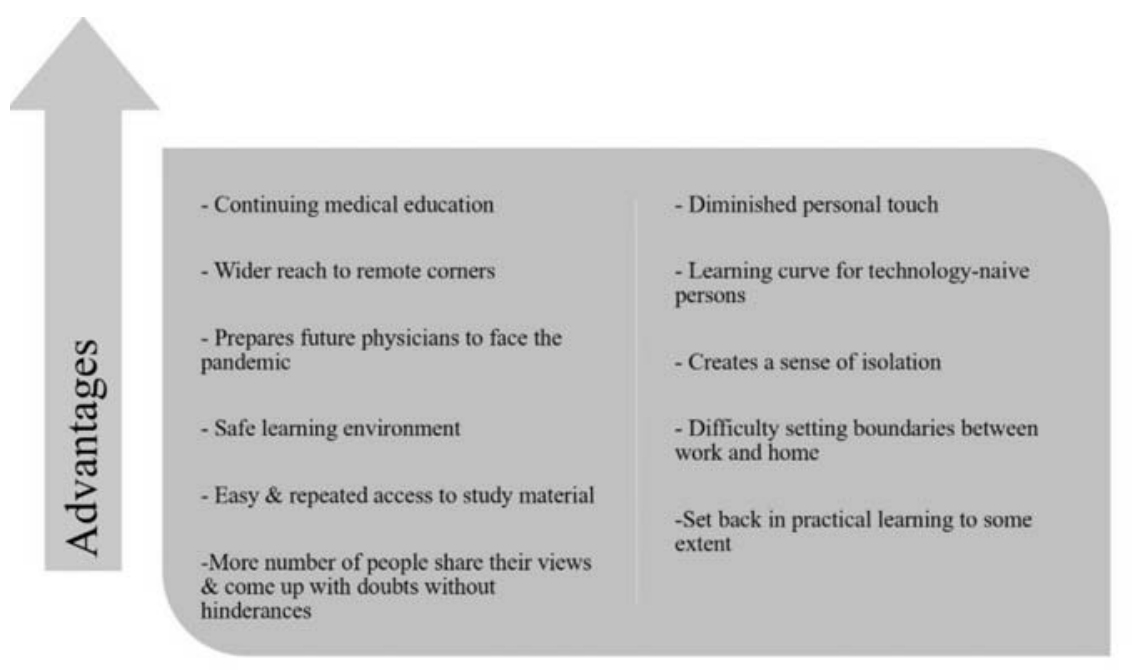

Fig. 3 Features of an online platform.

warmth, which was there during the physical meetings. To overcome the various problems, IAP has come up with specific solutions like facilitating 24-hour teaching modules with feedback for practical learning. The BLS (Basic life support) and ALS (Advanced Life Support) modules have been remodeled to suit e-learning. dIAP is also planning to include the live answers from the audience during the lectures through the e-notepad. With this software, the audience can easily select one of the options given by the speaker. This year of the pandemic will culminate with the highlight activity of IAP, i.e., the National PediWeek - dIAP will be conducting a virtual national conference which will take virtual teaching to a different level.

Charles Darwin said, "it is not the most intellectual of the species that survives; it is not the strongest that survives, but the species that survives is the one that is able best to adapt and adjust to the changing environment in which it finds itself." Online learning is the new way of life that we must adapt to in the days and weeks to come, which has been put into action by IAP.

I would like to personally thank Dr. Arun Bansal, Dr G V Basavaraj and Dr Namita Ravikumar who have spent their valuable time and effort to come up with the facts and figures pertaining to the different aspects in this document, making it an interesting and informative read. I also hope that we can achieve even greater heights by having a common goal - to enhance childcare in our country and ensure that IAP becomes a well-known organization, not just across the country, but rather across the entire world. Let us strive to make the best of these trying times and ensure the best for our mother organization.

Jai Hind! Jai IAP! 